\title{
"Comparative study on earthquake and ground based transmitter induced radiation belt electron precipitation at middle latitude", by Sideropoulos et al. (2011)
}

\author{
J.-A. Sauvaud ${ }^{1}$, M. Parrot ${ }^{2}$, and E. Slominska ${ }^{3}$ \\ ${ }^{1}$ IRAP-UMR5277, CNRS-University of Toulouse, Toulouse, France \\ ${ }^{2}$ LPC2E, UMR7328, CNRS-University of Orléans, Orléans, France \\ ${ }^{3}$ Space Research Center (CBK), Academy of Sciences, Warsaw, Poland \\ Correspondence to: J.-A. Sauvaud (jsauvaud@irap.omp.eu) \\ Received: 19 June 2013 - Published in Nat. Hazards Earth Syst. Sci. Discuss.: 26 July 2013 \\ Revised: 4 November 2013 - Accepted: 17 November 2013 - Published: 2 January 2014
}

\begin{abstract}
We show that many, if not all, electron bursts with energy dispersion claimed to be earthquake precursors by Sideropoulos et al. (2011) are due to the cyclotron resonance of electrons with monochromatic waves from VLF transmitters. The geographic distribution of the VLF-related electron bursts is established during a period in 2007, when the powerful NWC transmitter is off and 20 more transmitters are operating.
\end{abstract}

\section{Introduction}

Recently a number of papers have appeared in the literature claiming that earthquakes are preceded by the detection of electron bursts onboard the low altitude sun-synchronous satellite Demeter. Several of these papers present case studies and include some statistics (e.g., Sideropoulos et al., 2011, Anagnostopoulos et al., 2012), while others are based only on statistical studies (e.g., Zhang et al., 2010, 2013). While case studies allow a careful examination of the presented results, statistical studies are almost immune to examination of the initial data, allowing the authors to claim earthquake precursors using energetic particle data and VLF data. These works raise the question: Can precipitation bursts ever be uniquely identified as earthquake precursors?

The aim of this comment is to show that many, if not all, electron bursts with energy dispersion, measured along the orbit of Demeter and presented as an earthquake precursor by Sideropoulos et al. (2011), result from the cyclotron resonance of radiation belt electrons with monochromatic VLF waves emitted by powerful VLF transmitters. The paper is divided into two parts. In the first part we will examine bursts presented as examples of earthquake precursors when the NWC (North West Cape) transmitter in Australia is switched on. In the second part we will examine a published case pertaining to periods when NWC is switched off, i.e., from 1 July 2007 to 22 January 2008. During that period, geographical distributions of VLF transmitters and of the induced electron bursts are presented and modeled.

This study shows that the measured electron bursts showing a wisp structure are not associated with earthquakes, but with cyclotron resonance of electrons with monochromatic waves emitted by VLF transmitters.

Furthermore, enhancements in VLF activities recorded onboard Demeter have been claimed to be earthquake-related. We clearly show that these waves are linked to lightning activity. Moreover, it must be stressed that a large statistical study by Piša et al. (2013) with VLF Demeter data shows a decrease in the electric field at a frequency close to $1.7 \mathrm{kHz}$ a few hours before the earthquakes. This frequency is the usual cut-off frequency of the waves propagating in the Earth-ionosphere waveguide during nighttime, and this electric field is mainly due to the numerous whistler waves triggered by the thunderstorm activity. If the intensity of the electric field decreases, it means that the cut-off frequency is 
increasing, and then that the height of the ionosphere is statistically lower above epicenters. This indicates that the earthquake activity can change the propagation characteristics of VLF whistler waves, but no increase in the wave intensity is observed.

Finally, lightning-induced electron precipitation (LEP), i.e., electron bursts with very short duration $(<4 s)$ detected sometimes over atmospheric storms in association with whistler waves launched into the magnetosphere by lightnings will not be discussed in this paper (e.g., Rycroft, 1973; Voss et al., 1984; Inan et al., 1990, 2007; Gemelos et al., 2009; Parrot et al., 2013).

\section{NWC on}

In a recent paper, Anagnostopoulos et al. (2011) studied four cases of Demeter data showing electron bursts when the NWC transmitter was operating. It emits a power of $1 \mathrm{MW}$ at $19.8 \mathrm{kHz}$, in the North West Cape of Australia. The effect of this transmitter on energetic radiation belt electrons measured onboard the low-orbiting polar spacecraft DEMETER was reported by Sauvaud et al. (2008) and Gamble et al. (2008). More recently, a paper by Selesnick et al. (2013) provided a full simulation of the effect of the NWC waves on the radiation belt electrons based on a stochastic model of electron transport that includes pitch angle diffusion, radial diffusion, energy loss, and azimuthal drift.

The main signature of the interaction of energetic electrons with the transmitter monochromatic waves as seen at $700 \mathrm{~km}$ altitude is the formation of a wisp feature. The enhancements are initially observed within a few degrees west of NWC and are present in $95 \%$ of the orbital data east of the transmitter up to the South Atlantic Anomaly for time periods when the transmitter is broadcasting and located in nightside (Gamble, 2008; Sauvaud et al., 2008).

Studying electron bursts, Sideropoulos et al. (2011) did not take advantage of the fine energy resolution of the IDP energetic electron instrument onboard Demeter (see Sauvaud et al., 2006, 2013), and reached conclusions about electron burst based only on the examination of the variations along the Demeter orbit of three integral fluxes in the energy bands 92-526, 526-971 and 971-2350 keV (the energy range 72$90 \mathrm{keV}$, which is polluted by electronic noise, has been excluded). However, the energy-dispersed electron structure caused by NWC is only clearly apparent in the energy-time spectrograms of the IDP instruments, with 128 (256) energy channels in survey (burst) mode. In Fig. 1a and b we show such measurements, for two cases studied by Sideropoulos, together with the electric component of waves in the frequency range from $10 \mathrm{kHz}$ to $20 \mathrm{kHz}$ provided by the ICE instrument onboard Demeter and with the three integral energy fluxes discussed above. Figure 1a and b should be compared with Figs. 1a and 2a of Sideropoulos et al. (2011).

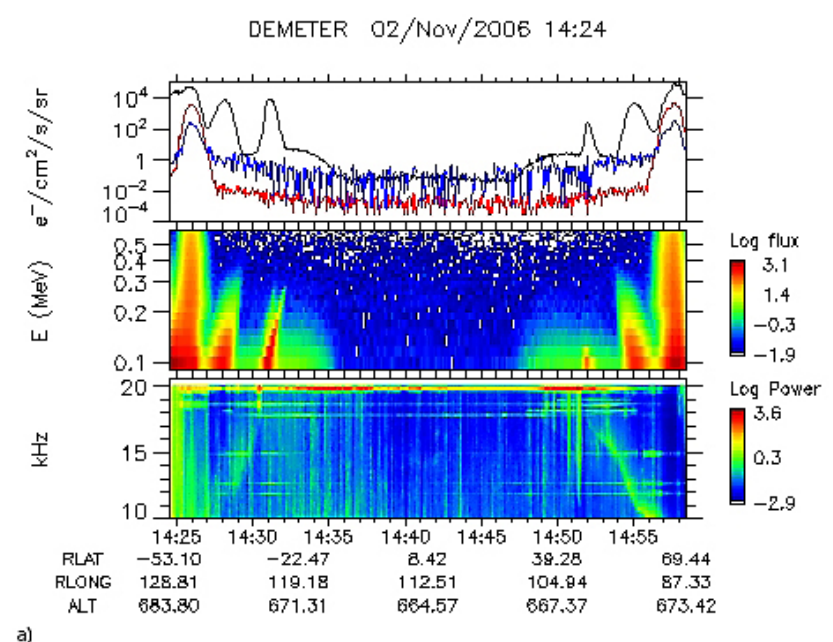

DEMETER $\quad 13 / \mathrm{Aug} / 2005 \quad 12: 53$

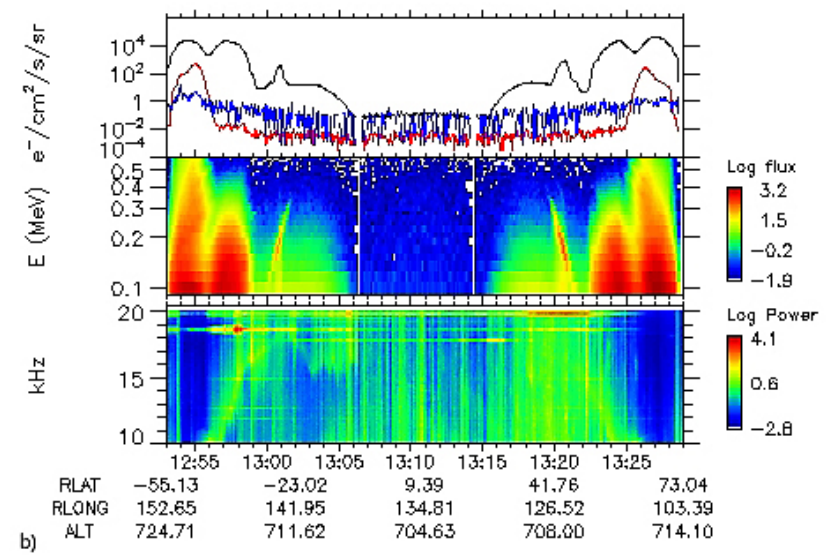

Fig. 1. (a) Energetic electrons and wave data along a half-orbit on 2 November 2006. The top panel gives the variations in the electron fluxes in three energy ranges $(90-526 \mathrm{keV}, 526-971 \mathrm{keV}$ and $971-$ $2350 \mathrm{keV}$ ). The middle panel shows the electron energy time spectrogram and the bottom panel gives the frequency-time spectrogram of the electric component of the VLF waves in the frequency range from 10 to $20 \mathrm{kHz}$. (b) Same as (a) for a pass on 13 August 2005.

Figure 1a presents measurements for which the electron bursts detected around 14:30 and 14:53 UT on 2 November 2006 have been attributed by Sideropoulos et al. (2011) to the near-equatorial resonance of electrons with the waves emitted by the NWC transmitter. The energy-time spectrogram of electrons presented in Fig. 1a indeed shows at 14:30 UT the typical energy-dispersed structure expected for such resonance, here in the Southern Hemisphere. Later during the same half-orbit, around 14:53 UT, the measurements are made slightly westward of the NWC transmitter, which results in a less marked structure.

Figure $1 \mathrm{~b}$ presents similar measurements performed over Australia east of NWC on 13 August 2005 between 09:14 and 09:53 UT, during a pass considered as earthquake related by Sideropoulos et al. (2011). Demeter indeed encounters 


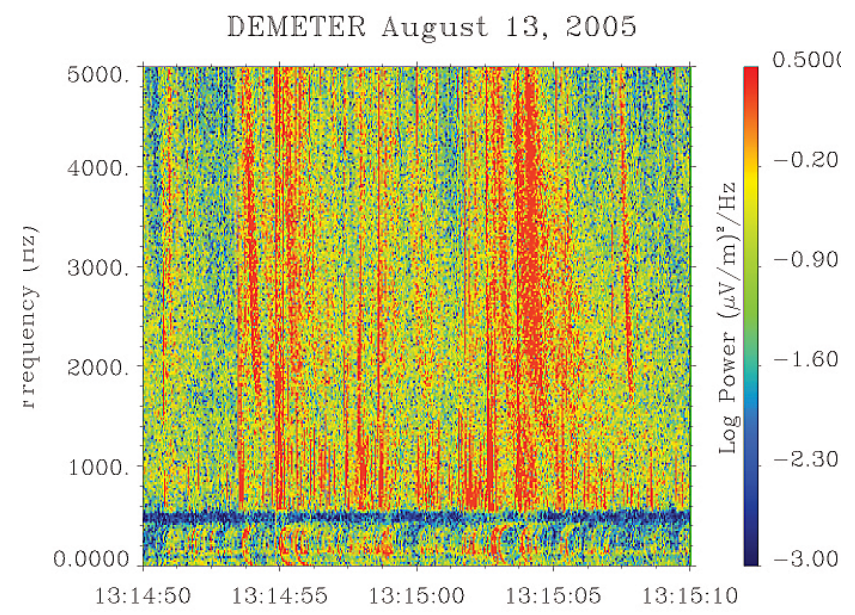

Fig. 1c. Detailed frequency-time spectrogram of the electric component of the waves in the $0-5000 \mathrm{~Hz}$ range measured onboard Demeter from 13:14:50 and 13:15:10 UT on August 13 2005, when Demeter is in burst mode (see Fig. 1b). Whistlers with frequencytime dispersion can be clearly distinguished. Their dispersion is however small, which comes from the fact that lightnings occur below the spacecraft, in the same hemisphere. Below the local proton gyro frequency $(500 \mathrm{~Hz})$, a number of proton whistlers, also related to lightnings, are visible. Using the WWLLN lightnings database, it was verified that Demeter encountered a number of lightnings over a very large region of thunderstorms during this northern pass over Asia.

electron bursts two times, at conjugate locations in the Northern and Southern hemispheres, around 13:01 and 13:20 UT. It must be stressed that the energy-dispersed nature of the bursts is clearly visible in Fig. $1 \mathrm{~b}$ and that they are very similar to the structures displayed in Fig. 1a. However, Sideropoulos et al. (2011) attributed these bursts to a "clear example" of a precursor to an earthquake occurring on 16 August 2005 in Japan. They associated the electron burst with the enhanced VLF signal recorded in the Northern Hemisphere between 13:17 and 13:25 UT and noted that another VLF burst was also seen in the Southern Hemisphere. Two main remarks must be made regarding this association: (i) the considered VLF waves being largely distributed in frequency, they should resonate with electrons in a broad range of energies, which is clearly not observed, and (ii) the power of the natural VLF waves is weak compared with the NWC signal. In order to illustrate this fact, Fig. 2 presents the nightside distribution of the VLF waves in a frequency range $15-25 \mathrm{kHZ}$ in the vicinity of Australia and in the conjugate hemisphere. Just above NWC the average wave power is above $10^{4} \mu \mathrm{V}^{2} \mathrm{~cm}^{-2} \mathrm{~s}$, while the power of the natural VLF waves recorded onboard Demeter around 13:20 UT is less than $10 \mu \mathrm{V}^{2} \mathrm{~cm}^{-2} \mathrm{~s}$. The effect of NWC is thus expected to be completely dominant.

The measured VLF waves in Fig. $1 \mathrm{~b}$ are examined in detail as in Fig. 1c. These emissions are made up of discrete packets
$19.8 \mathrm{kHz}$

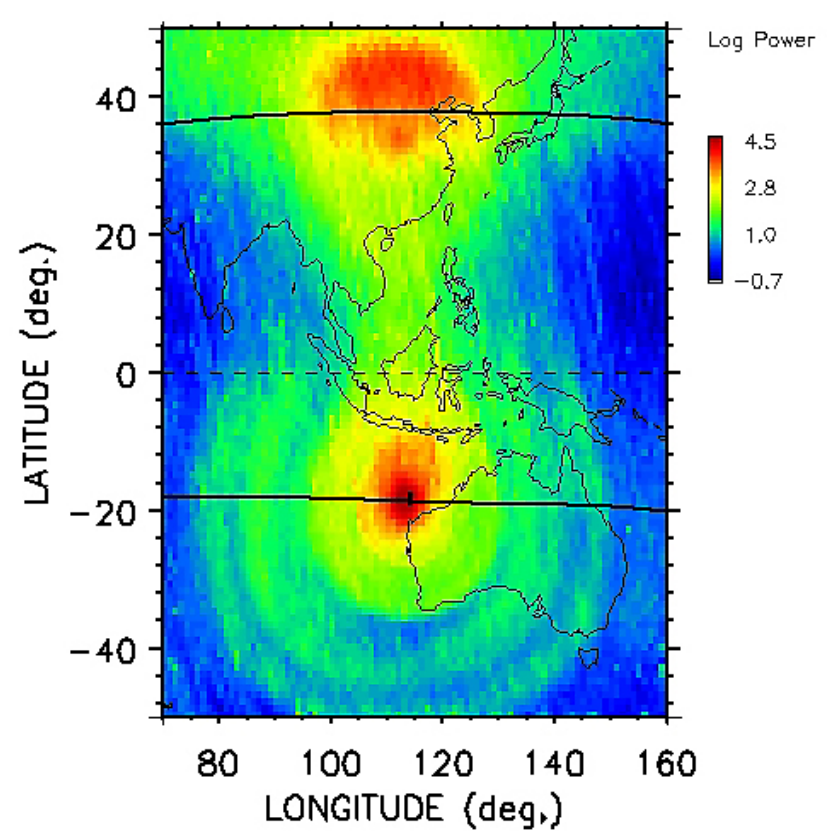

Fig. 2. Distribution of the electric power of the NWC transmitter at Demeter altitudes. The power of the NWC transmitter is $10^{4}$ higher than that measured at 13:20 UT on 13 August 2005 in the frequency range $10-19 \mathrm{kHz}$ (see Fig. 1). The black curves indicate the $L=1.4$ contours at the satellite altitude.

of whistler waves, measured when Demeter is in burst mode. At the lowest frequencies, below the local proton gyro frequency, proton whistlers are detected. They are known to occur immediately after an upwardly propagating whistler has been generated by a lightning discharge. We have been able to check, using the WWLLN database, that Demeter passes over a large region where numerous lightnings occur in the Asian sector. It is thus natural to attribute the VLF signal detected by Demeter to the effect of lightnings instead of invoking a hypothetical precursor to an earthquake (see also Inan et al., 2007; Parrot et al., 2013).

Furthermore, the electron bursts, when displayed in a energy-time spectrogram, show the typical dispersion structure resulting from their interaction with monochromatic waves. Recently, Selesnick et al. (2013) performed a full simulation of the effect of the NWC waves on the radiation belt electrons based on a stochastic model of electron transport that includes pitch angle diffusion, radial diffusion, energy loss, and azimuthal drift. They showed that the Demeter electron-dispersed structures are in agreement with their calculations.

More simply, we can compute the energy of resonant electrons with $19.8 \mathrm{kHz}$ waves. The results are given in Fig. 3, where we display the electron-dispersed structure measured at around 13:20 UT on 13 August 2005, together with the 


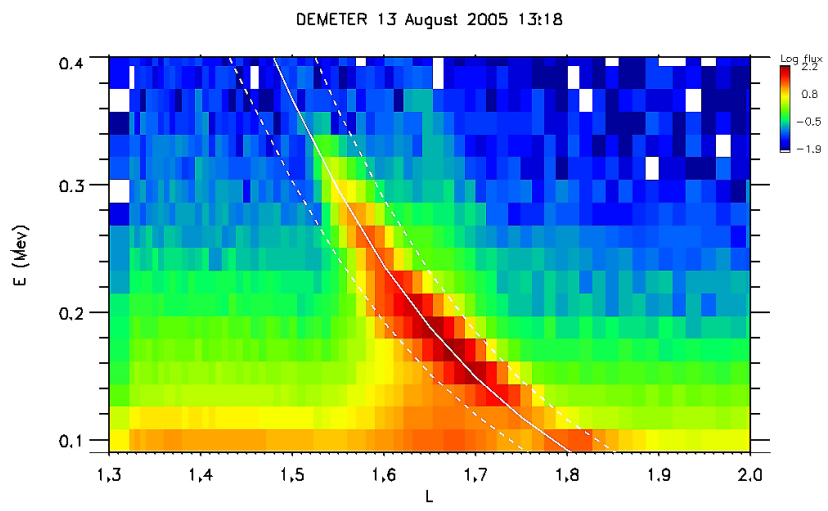

Fig. 3. Electron energy $L$ spectrogram for the structure recorded on 13 August 2005 starting at 13:18 UT. The white line is the result of the computation of wave-particle interaction described in the text. The dashed lines are for a plasmaspheric plasma density enhanced (lower curve) and divided by a factor 1.3 (upper curve).

energy change expected from a simple model of equatorial cyclotron resonance.

Whistler-mode waves from NWC are at fixed-frequency $\omega$, with the index of refraction $\mu=k c / \omega$ given by

$\mu^{2}=1+\frac{\omega_{\mathrm{p}}^{2}}{\omega(\Omega|\cos \theta|-\omega}$

for wave number $k$, wave normal angle $\theta$, plasma frequency $\omega_{\mathrm{p}}$, and electron cyclotron frequency $\Omega$. The condition for electron-wave cyclotron resonance is

$\omega-k_{\|} v_{\|}=\frac{n \Omega}{\Upsilon}$,

where $n$ is a positive or negative integer, or zero, $k_{\|}=k \cos \theta$, $v_{\|}=v \cos \alpha, v$ is the electron speed, $\gamma$ the Lorentz factor, and $\alpha$ is the local pitch angle. The wave normal is taken equal to $60^{\circ}$. For equatorial resonance, the pitch angle of electrons is computed in a way that electrons have a $90^{\circ}$ pitch angle at the satellite altitude. Following Selesnick et al. (2013), for evaluation of the plasma frequency, the plasma density is taken equal to $A \times 10^{3} \mathrm{~L}^{-1.7} \mathrm{~cm}^{-3}$, with A varying in the range $9000-18000 \mathrm{~cm}^{-3}$. Note that the ionospheric density has been neglected, as above $L=1.4$ it represents only a weak part of the plasmaspheric one.

The results of the computations are displayed together with the measured electron energy spectrogram (Fig. 3). The computed energy resonance (white curves) well fit the measured electron dispersion shape. Here the A term in the plasma density relation is taken equal to $18000 \mathrm{~cm}^{-3}$ for the solid curve. The dashed curves are for an inner plasmaspheric density $30 \%$ higher and $30 \%$ lower.

From the comparison of the shape and location of the energy structures and of the model energy band we conclude, following earlier works by Sauvaud et al. (2008), Gamble et al. (2008) and Selesnick et al. (2013), that the observed "electron burst" is due to the resonance of electrons in the inner radiation belt drift with the monochromatic wave emitted by the NWC transmitter, followed by their eastward drift and can hardly be interpreted as being due to a hypothetical precursor of the Japan earthquake occurring days after the observations.

Figure $4 \mathrm{a}$ and $\mathrm{b}$ present two more cases of Demeter measurements close to the Australian NWC meridian on 12 May 2006 and 16 May 2006. These figures must be compared with Fig. 4a and b of Sideropoulos et al. (2011). The measurements performed onboard Demeter on May 122006 (Fig. 4a) are similar to those presented in Fig. 1; the spacecraft passes eastward of NWC, i.e., in a region where the electrons drift, and the same interpretation applies there. Furthermore, note in Fig. 4a that around 10:58 UT, besides the wisp structure, there is a weak vertical feature. It corresponds to the highest fluxes in the wisp structure itself (around $160 \mathrm{keV}$ ). Checking other wisps all around the Earth, we found several of these weak vertical structures, each time associated with the highest fluxes inside the wisps. The signal at the lowest energies is due to the interaction of the wisp electrons with the collimator of the spectrometer; the resulting low energy population has indeed been measured during calibration in the accelerator, when $200 \mathrm{keV}$ electrons are sent to the detector (Moreau, 2006). The high-energy counterpart is due to event pile-up at the charge pre-amplifier. Note that in Fig. 1a, the same effect is seen although less marked for the event at 14:31-14:32 UT, while this event according to Anagnostopoulos et al. (2011) is not associated with an earthquake.

Furthermore, the intensification of VLF activity seen between 10:32 and 10:45 UT in Fig. 4a (and between 09:17 and 09:25 in Fig. 4b) has been studied as in the case presented in Fig. 1b. The burst data once again clearly show that whistlers associated with atmospheric lightnings are at the root of the VLF observations.

As stated in Sideropoulos et al. (2011), Fig. 4b shows no electron burst around 09:25 UT. At NWC, the sunset is at 17:50 LT, which corresponds to 09:50 UT. Furthermore, accounting for the drift time of $100 \mathrm{keV}$ electrons between NWC and the satellite location adds a delay of about $1 \mathrm{~h}$. Electrons should have thus left the NWC location at 08:25 UT, i.e., 16:25 LT, i.e., during daylight. At 16:25 LT, NWC is still in the day and the waves are strongly attenuated by the ionosphere (Cohen and Inan, 2012). It is thus expected that no clear electron structure resulting from wave-particle interaction can be detected. Later, when the spacecraft reach the conjugate hemisphere, a very weak structure can be seen in Fig. 4b around 09:43 UT in the energy-time electron spectrogram for energies of about $300 \mathrm{keV}$. The transmitter is now in penumbra and a weak part of the wave power escapes the ionosphere and interacts with electrons.

To conclude this part, the absence of well-marked energydispersed structure is here due to the local time of the NWC station and cannot be attributed to the fact that "electron 

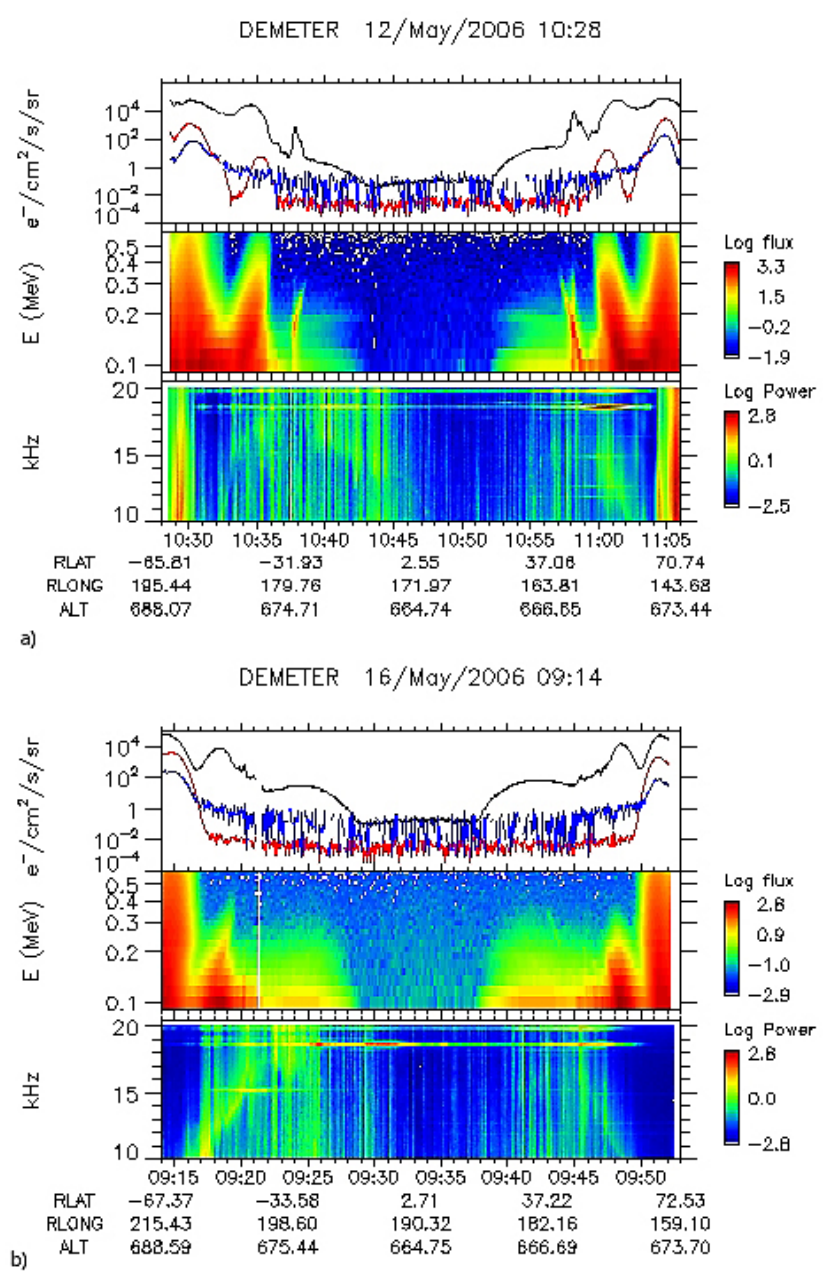

Fig. 4. (a) Electron and wave (see Fig. 1) measurements on 12 May 2006 between 10:26 and 11:06 UT. Conjugate electron wisps are detected around 10:38 and 10:58 UT. (b) Same as (a) for 16 May 2006. This figure must be compared with Figs. 3 and 4 of Sideropoulos et al. (2011).

precipitation activity ceases a few hours before a great earthquake", as stated in Sideropoulos et al. (2011).

\section{NWC off}

Sideropoulos et al. (2011) also studied electron burst when the NWC transmitter is off. They presented a case (their Fig. 3) obtained on 18 September 2006 where two electron bursts are detected.

Figure 5 gives the Demeter measurements during that period in the same format as in Figs. 1a, b and 4a, b. A slightly dispersed electron structure is clearly seen at low energies around 13:14-13:15 UT. A conjugate enhancement in electron fluxes is seen at the lowest energies around 12:5312:54 UT. Such electron flux enhancements are quite common during the period when NWC was off, i.e., between

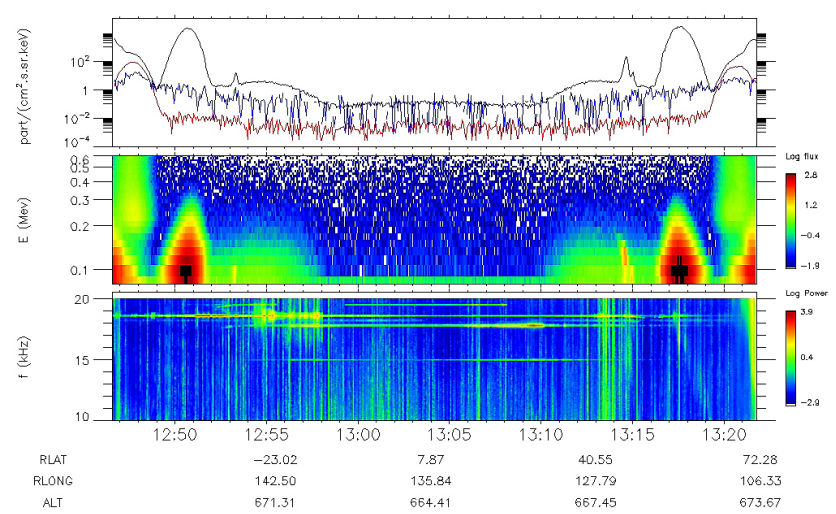

Fig. 5. Same format as Figs. 1 and 4, for 18 September 2007 between 12:46 and 13:22 UT, when NWC was off.

1 July 2007 and 22 January 2008. Before claiming an earthquake cause for such measurements, one has to check if these bursts can be produced from the interaction of electrons with other VLF transmitters.

The geographic distribution of the VLF transmitter is given in Figs. 6, 7 and 8. Figure 6 displays the power distribution measured at Demeter altitude between 15 and $25 \mathrm{kHz}$ (see Parrot et al., 2009). In Fig. 6 the black lines indicate the $L=2$ contours in the Northern and Southern hemispheres. In the US sector, four main transmitters are operating: NPM at very low latitude, and NLK, NML and NAA at $L$ values somewhat higher than 2 . The emitted waves can be seen in the Southern Hemisphere around $L=2$. It means that if electrons resonate with these transmitters, the energy-dispersed structures should be detected at an $L$ value of the order of 2 . In Europe three main transmitters are detected: GQD in UK, HWU in France and DHO in Germany; in the south hemisphere, the corresponding waves are detected southward of South Africa around $L=2$. In the Asian sector three transmitters are detected, in the Southern Hemisphere NWC at $L=1.4$ and NTS at $L$ slightly higher than 2. In the Northern Hemisphere: UBE at $L$ equal to 2 is confused with the conjugate waves of NTS. Except for NWC, all the transmitters were operating during the period when the data presented in Fig. 5 were obtained.

Furthermore, other navigation transmitters work at lower frequencies. Figure 7 gives the position of three ALFA transmitters in Russia, which emit sequentially at $11.8 \mathrm{kHz}$, $12.6 \mathrm{kHz}$ and $14.8 \mathrm{kHz}$. Figure 8 provides measurements at higher frequencies, between 25 and $60 \mathrm{kHz}$. Here 12 more transmitters are clearly identified. Two of them are located just below $L=1.5$ (NAU (US) and NSC (Italy)), with their conjugate area around $L=1.5$. Note that in Figs. 6 and 8 the frequency resolution is only $3.2 \mathrm{kHz}$, which leads to a large underestimation of the measured power of the transmitters, which emits nearly monochromatic waves. Table 1 provides the transmitters' codes and geographical position. 
Table 1. Locations of the VLF transmitters displayed in Figs. 6, 7 and 8.

\begin{tabular}{lrrr}
\hline $\begin{array}{l}\text { Call } \\
\text { signs }\end{array}$ & $\begin{array}{r}\text { Frequency } \\
(\mathrm{kHz})\end{array}$ & $\begin{array}{r}\text { Latitude } \\
\left({ }^{\circ}\right)\end{array}$ & $\begin{array}{r}\text { Longitude } \\
\left({ }^{\circ}\right)\end{array}$ \\
\hline KRA & 11.90 & 45.50 & 38.10 \\
NOV & 12.65 & 55.60 & 84.40 \\
KOM & 14.88 & 50.32 & 136.59 \\
UBE & 16.20 & 52.90 & 158.55 \\
HWU & 18.30 & 46.70 & 01.23 \\
NST & 18.60 & -38.48 & 146.93 \\
GQD & 19.60 & 52.91 & -3.28 \\
NWC & 19.80 & -21.82 & 114.17 \\
HWV & 20.90 & 48.54 & 02.52 \\
NPM & 21.40 & 21.42 & -158.15 \\
NDT & 22.20 & 32.08 & 130.83 \\
DHO & 23.40 & 53.08 & 07.61 \\
NAA & 24.00 & 44.65 & -67.29 \\
NLK & 24.80 & 48.20 & -121.92 \\
TBB & 26.70 & 37.43 & 26.70 \\
JJY-40 & 40.00 & 37.37 & 140.85 \\
NAU & 40.80 & 40.80 & -67.18 \\
NSC & 45.90 & 38.00 & 13.50 \\
NML & 46.37 & 46.36 & -98.34 \\
WWVB & 60.00 & 40.56 & -105.07 \\
JJY-60 & 60.00 & 33.46 & 130.17 \\
\hline & & & \\
\hline
\end{tabular}

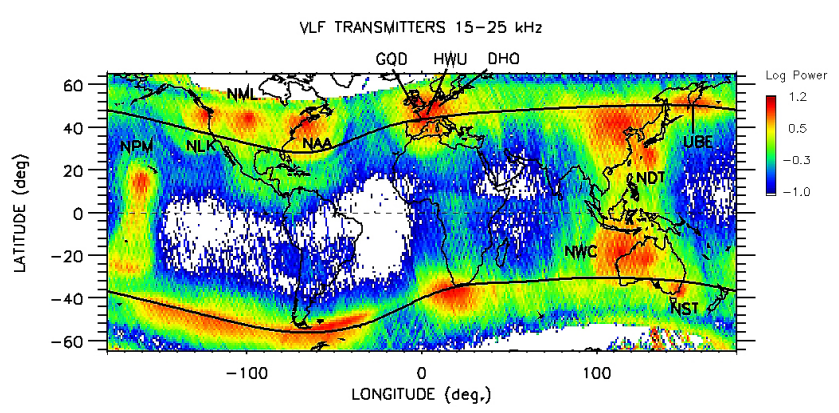

Fig. 6. Average distribution of electric power in the $15-25 \mathrm{kHz}$ range measured during night passes during $3 \mathrm{yr}$ (2007-2009). The corresponding transmitters are indicated by their international code. The two black lines indicate the $L=2$ contours in the Northern and the Southern hemispheres.

The result of a search for dispersed electron structure during a three-month period from July to September 2007, when NWC was off, is presented in Fig. 9. The upper panel shows the distribution of the electron bursts in a UT-longitude diagram. As expected from the sun-synchronous orbit of Demeter, the bursts are found along straight lines resulting from the rotation of the Earth below the satellite. UT is always $\mathrm{UT}=10$-long $/ 15$ and UT $=22$-long/15. Red symbols are for measurements made near 10:00 LT and black ones for measurements made near 22:00 LT. The black (red) double traces correspond to nightside (dayside) passes of the spacecraft crossing first a northern structure and about one hour later the
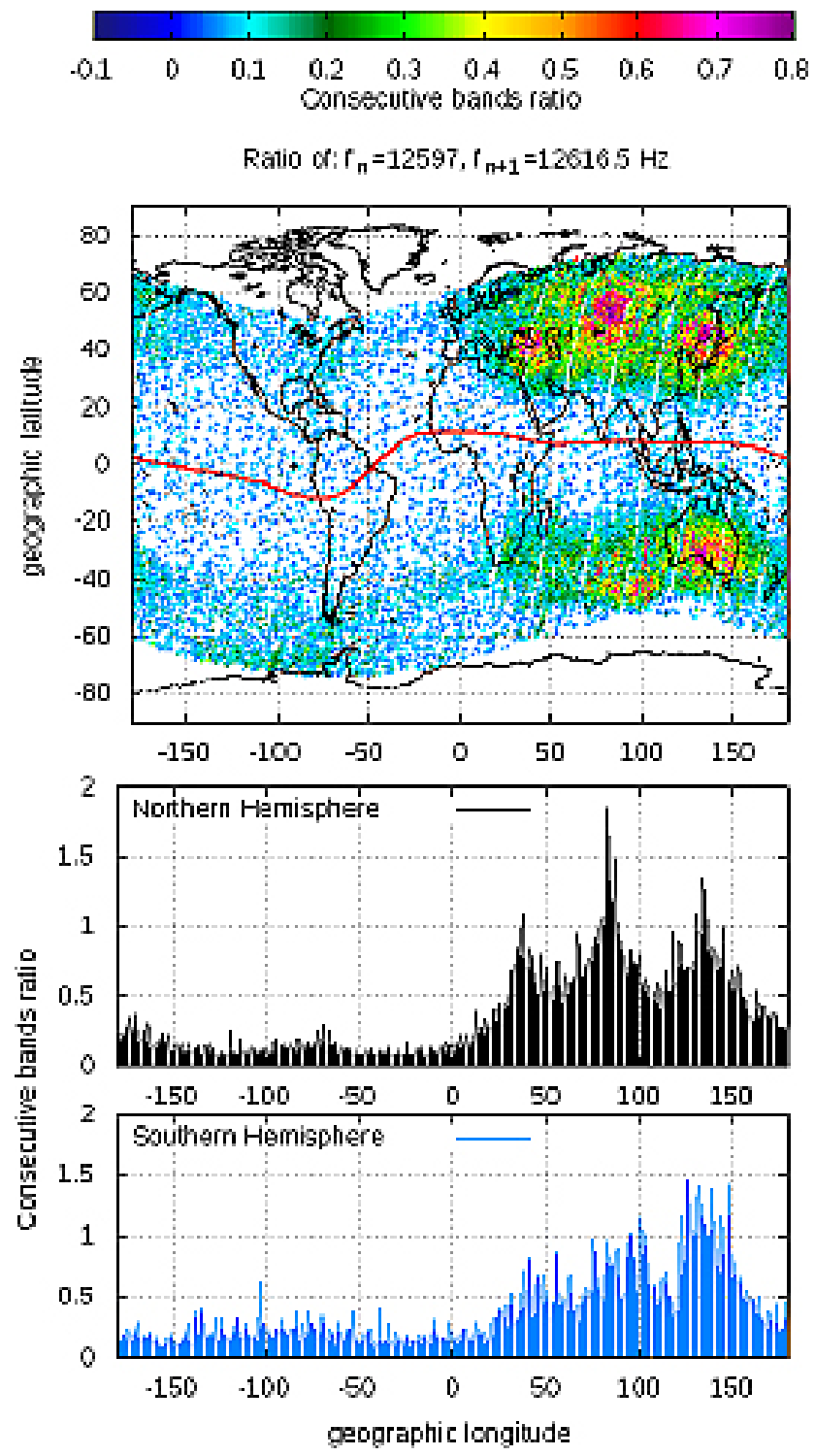

Fig. 7. Top panel: position of the three Russian transmitters emitting in the Northern Hemisphere at $11.8,12.8$ and $14.8 \mathrm{kHz}$. Middle panel: consecutive frequency band ratio for the Northern Hemisphere. Bottom panel: same for the Southern Hemisphere.

conjugate structure in the Southern Hemisphere, after flying over the dayside (nightside) part of the Earth. Single traces correspond to structures with no conjugate. The structures are thus well organized in a UT-longitude space, indicating a probably geographic origin. The bottom panel of Fig. 9 gives the latitude-longitude distribution, with superposed curves giving the $L=2$ and $L=1.5$ locations in the Northern and Southern hemispheres. The structures are mainly grouped along $L=2$, with two notable exceptions: in the US sector at longitudes from -100 to $-70^{\circ}$ and in the South African sector at longitudes between 10 and $40^{\circ}$, where they are located at lower $L$ shells. 


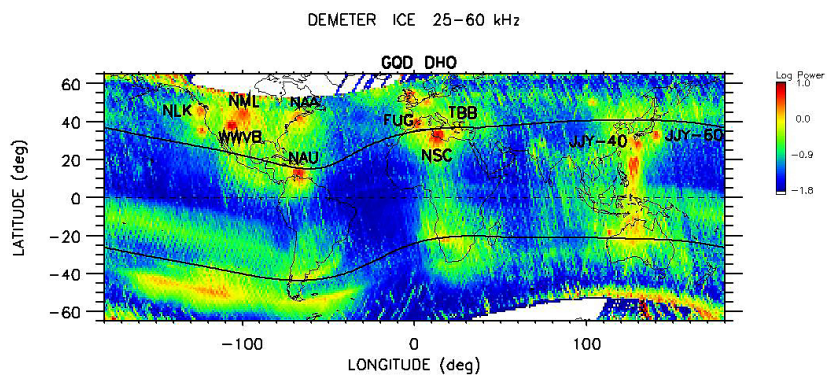

Fig. 8. Average distribution of electric power in the $25-60 \mathrm{kHz}$ range measured during night passes during $3 \mathrm{yr}$. The corresponding transmitters are indicated by their international code. The two black lines indicate the $L=1.5$ contours in the Northern and the Southern hemispheres.

Let us suppose that each structure is resulting from the interaction of electrons with VLF waves emitted by human transmitters. For the waves to reach the magnetosphere, the station must be in darkness or at the beginning of the day, in order for the electron density in the D-region of the ionosphere not to be high enough to prevent the escape of VLF power inside the magnetosphere (Cohen and Inan, 2012). Taking this into account it becomes simple to find the origin of the structures. Their origins are indicated in the upper panel of Fig. 9. US stand for United States, EU for Europe. Russia and Asia are also indicated. There are no structures recorded over the Atlantic; this comes from the fact that there the electrons drifting from the other part of the Earth are precipitating, due to the lowering of their mirror point in the Southern Hemisphere.

It is also remarkable that nightside passes over Europe do not show electron structures, while in the conjugate region there are a number of recorded electron structures. We attribute this to the weak field in the South African sector, located on the eastern side of the South Atlantic Anomaly, where electrons resonating with the waves emitted by the European transmitter can be detected in the low B-field region at the satellite altitude. To illustrate this interpretation, we show in Fig. 10 a pass over South Africa where three successive electron bursts are recorded. The most probable causes of the two structures at the highest $L$ shells are the French FUG and Turkish TBB transmitters, respectively emitting at 22.67 and $21.75 \mathrm{kHz}$, while the Italian NSC station causes the electron wisp, at lower $L$ shell. Note in Fig. 10 that there is a similar effect at a longitude of about $-100^{\circ}$, where the electrons emitted by the low-latitude NAU transmitter are only detected in the Southern Hemisphere, at the western border of the South Atlantic Anomaly.

Finally, Fig. 9 clearly shows that the location of the electron bursts (green symbols) detected as an earthquake precursor by Sideropoulos et al. (2011) are exactly superposed on the traces of electron bursts due to the cyclotron resonance of electrons with VLF transmitters.

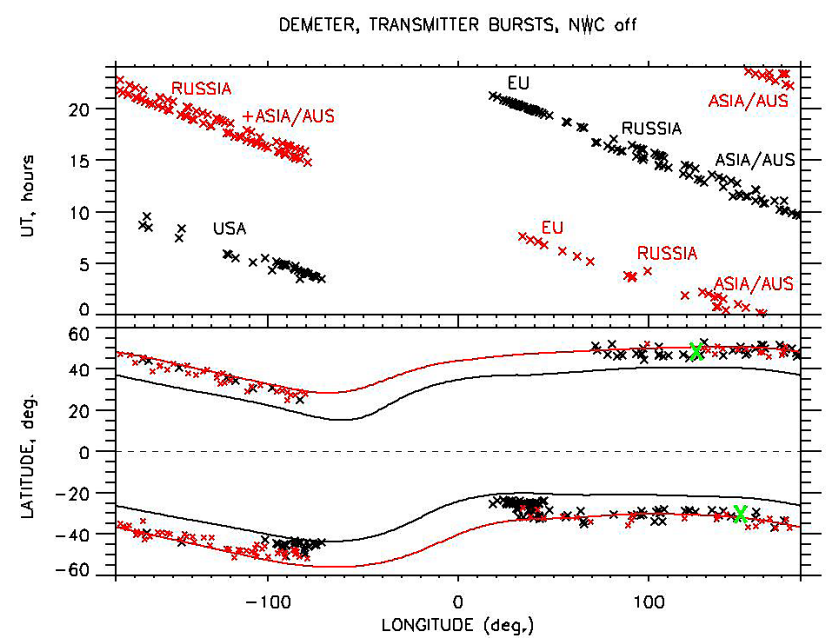

Fig. 9. UT-longitude (top) and latitude-longitude distribution of electron burst during the three months (July-August, September 2007) when the NWC transmitter is off. The red symbols indicate measurements made around 10:00 local time, while the black symbols are for 22:00 local time. The red and black curves in the bottom panel show respectively the $L=2$ and $L=1.5$ locations in the Northern and Southern hemispheres. The green crosses correspond to the measurement of electron bursts presented in Sideropoulos et al. (2011) as due to a precursor to an earthquake. The regions where the bursts are produced are indicated in the top panel.

$$
\text { DEMETER } \quad 45900-26700-21750 \mathrm{~Hz} \quad 25 / \mathrm{Sep} / 2007 \text { 19:39 }
$$

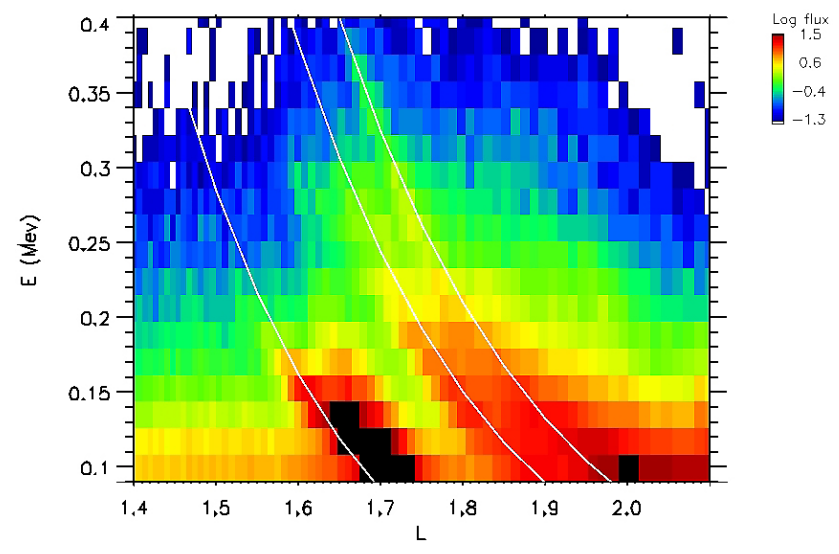

Fig. 10. Multiple electron wisps resulting from the cyclotron interaction of human VLF with electrons from the inner belt. Computed energy dispersions of electrons resonating with monochromatic waves emitted at 45900,26400 and $21750 \mathrm{~Hz}$ are shown by the white curves.

\section{Discussion and conclusion}

We have presented evidence for the association of electron bursts recorded onboard Demeter with VLF waves of human origin. The powerful NWC transmitter has the most pronounced effects on the inner radiation belt electron 
population, as demonstrated in several papers (e.g., Sauvaud et al., 2008; Gamble et al., 2008; Li et al., 2011). Measurements made eastward of NWC, i.e., along the drift trajectory of electrons, should not be attributed to another cause without a very careful check of the transmitter expected effect. When this transmitter is in daylight, the absence of electron wisp should first be related to the absorption of the waves by the dayside ionosphere.

When the NWC transmitter is off, 20 more transmitters are still operating, with a clear effect of diffusing a part of the inner belt electrons inside the drift loss cone. A statistical study of 3 months of data taken during such a period shows that the resulting "electron bursts" are distributed worldwide. Electron bursts, well fitted by electron-VLF wave resonance models, are indeed seen at all longitudes, except at those corresponding to the South Atlantic Anomaly.

A careful examination of the cases presented in Sideropoulos et al. (2011) as earthquake precursors thus indicate that all bursts containing wisp structure are consistent with being caused by ground transmitters. Furthermore, VLF intensifications observed over large regions with a power much lower than that of the NWC transmitter appear to be due to lightnings. Note also that electron bursts at low energies $(E<200 \mathrm{keV})$ without wisps have been registered in association with whistler waves generated by lightnings (Inan et al., 2007; Gemelos et al., 2009).

The results presented here shed some doubt on earthquake studies performed with the Demeter data without exploring the possibility that electron bursts could be linked with VLF transmitters (Anagnostopoulos, 2011; Sideropoulos et al., 2011; Zhang et al., 2010, 2013). Furthermore, the fact that VLF transmitters are nearly continuous from many locations, whereas large earthquakes occur only occasionally, places a heavy burden of justification on any interpretation of electron bursts favoring earthquakes.

\section{Supplementary material related to this article is available online at http://www.nat-hazards-earth-syst-sci.net/14/1/2014/ nhess-14-1-2014-supplement.pdf.}

Acknowledgements. The authors thank the referees for helpful suggestions and comments on the dispersed nature of electron bursts. Data display software has been provided by Emmanuel Penou (http://clweb.cesr.fr/).

Edited by: F. Masci

Reviewed by: R. Selesnick and Y. Chen

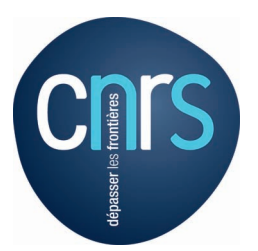

The publication of this article is financed by CNRS-INSU.

\section{References}

Anagnostopoulos, G. C., Vassiliadis, and Pulinets, S.: Characteristics of flux-time profiles, temporal evolution, and spatial distribution of radiation-belt electron precipitation bursts in the upper ionosphere before great and giant earthquakes, Ann. of Geophysics, 55, 2012; doi:10.4401/ag-5365, 2011.

Cohen, M. B. and Inan, U. S.: Terrestrial VLF transmitter injection into the magnetosphere, J. Geophys. Res., 117, A08310, doi:10.1029/2012JA017992, 2012.

Cohen, M. B., Lehtinen, N. G., and Inan, U. S.: Modems of ionospheric VLF absorption of powerful ground based transmitters, Geophys. Res. Lett., 39, L24101, doi:10.1029/2012GL054437, 2012.

Gamble, R. J., Rodger, C. J., Clilverd, M. A., Sauvaud, J. A., Thomson, N. R., Stewart, S. L., Mccormick, R. J., Parrot, M., and Berthelier, J.-J.: Radiation belt electron precipitation by man-made VLF transmissions, J. Geophys. Res., 113, A10211, doi:10.1029/2008JA013369, 2008.

Gemelos, E. S., Inan, U. S., Walt, M., Parrot, M. and Sauvaud, J.A.: Seasonal dependence of energetic electron precipitation, Evidence for a global role of lightning, Geophys. Res. Lett., 36, L21107, doi:10.1029/2009GL040396, 2009.

Koons, H. C., Edgar, B. C., and Vampola, A. L.: Precipitation of inner zone electrons by whistle-mode waves from VLF transmitters UMS and NWC, J. Geophys. Res., 86, 640-648, 1981.

Li, X., Yugian, M., Wang, P., Wang, H., Lu, H., Zhang, X., Huang, J., Shi, F., Yu, X., Xu, Y., Meng, X., Wang, H., Zhao, X., and Parrot, M.: Study of the North West Cape electron belts observed by DEMETER satellite, J. Geophys. Res., 117, A04201, doi:10.1029/2011JA017121, 2012.

Moreau, T.: Etude numérique et développement de spectromètres embarquables de particules chargées, Ph.D. Thesis, University Paul Sabatier, Toulouse, 2003.

Parrot, M., Inan, U. S., Lehtinen, N. G., and Pinçon, J. L. Penetration of lightning MF signals to the upper ionosphere over VLF ground-based transmitters, J. Geophys. Res., 114, A12318, doi:10.1029/2009JA014598, 2009.

Parrot, M., Sauvaud, J. A., Soula, S., Pinçon, J. L., and Velde, O.: Ionospheric density perturbations recorded by DEMETER above intense thunderstorms, J. Geophys. Res., 118, 5169-5176, doi:10.1002/jgra.50460, 2013.

Piša, D., Němec, F., Santolik, O., Parrot, M., and Rycroft M.: Additional attenuation of natural VLF electromagnetic waves observed by the DEMETER spacecraft resulting from preseismic activity, J. Geophys. Res.-Space, 118, 5286-5295, doi:10.1002/jgra.50469, 2013.

Sauvaud, J. A., Moreau, T., Maggiolo, R., Treilhou, J.-P., Jacquey, C., Cros, A., Coutelier, J.-J., Rouzaud, J., Penou, E., and Gangloff : M., High-energy electron detection onboard DEMETER: The IDP spectrometer, description and first results on the inner belt, Planet. Space. Sci., 54, 502-511, 2006.

Sauvaud, J.-A., Maggiolo, R., Jacquey, C., Parrot, M., Berthelier, J.J., Gamble, R. J., and Rodger, C. J.: Radiation belt electron precipitation due to VLF transmitters: Satellite observations, Geophys. Res. Lett., 35, L09101, doi:10.1029/2008GL033194, 2008.

Sauvaud, J.-A., Walt, M., Delcourt, D., Benoist, C., Penou, E., Chen, Y., Russell, C. T.: Inner radiation belt particle acceleration and energy structuring by drift resonance with ULF waves 
during geomagnetic storms, J. Geophys. Res., 118, 1723-1736, doi:10.1002/jgra.50125, 2013.

Selesnick, R. S., Albert, J. M., and Starks, M. J.: Influence of ground-based VLF radio transmitter on the inner electron radiation belt, J. Geophys. Res., 118, 628-635, doi:10.1002/jgra.50095, 2013.

Sidiropoulos, N. F., Anagnostopoulos, G., and Rigas, V.: Comparative study on earthquake and ground based transmitter induced radiation belt electron precipitation at middle latitudes, Nat. Hazards Earth Syst. Sci., 11, 1901-1913, doi:10.5194/nhess-111901-2011, 2011.
Zhang, X., Fidani, C., Huang, J., Shen, X., Zeren, Z., and Qian, J.: Burst increases of precipitating electrons recorded by the DEMETER satellite before strong earthquakes, Nat. Hazards Earth Syst. Sci., 13, 197-209, doi:10.5194/nhess-13-197-2013, 2013.

Zhang, Z., Li, X., Wu, S., Ma, Y., Shen, X., Chen, H., Wang, P., You, X., and Yuan, Y.: DEMETER, satellite observations of energetic particle prior to Chile earthquake, Chinese J. Geophys., 5, 15811590, English version at: arXiv:1011.3592v1, 2010. 